\title{
Polishing, chemical etching and thermal treatment effects on surface and electrical properties of Er and Nd-doped GaSb substrates
}

\author{
J.L. Plaza ${ }^{\mathrm{a}, *}$, P. Hidalgo ${ }^{\mathrm{b}}$, B. Méndez ${ }^{\mathrm{b}}$, J. Piqueras ${ }^{\mathrm{b}}$, E. Diéguez ${ }^{\mathrm{a}}$ \\ a Departamento de Física de Materiales, Universidad Autónoma de Madrid 28049, Cantoblanco, Madrid, Spain \\ ${ }^{\mathrm{b}}$ Departamento de Física de Materiales, Universidad Complutense 28040, Madrid, Spain
}

\begin{abstract}
In this work several changes induced by polishing, chemical etching and thermal treatment in the properties of Er- and Nd-doped GaSb substrates with different doping levels grown by the vertical Bridgman method have been studied. The analysis has revealed the formation of precipitates at the highest doping levels for both Er- and Nd-doped GaSb. Cathodoluminescence analysis shows the reduction of the defect band induced by the rare earth (RE) elements. For high dopant concentrations the precipitation phenomena reduce this effect. Thermal treatments have produced an enhancement of the p-type properties of the samples. For a dopant density of $2 \times 10^{19} \mathrm{~cm}^{-3}$ this effect is more pronounced. (C) 2000 Elsevier Science S.A. All rights reserved.
\end{abstract}

Keywords: Gallium antimonide; Rare earths; Thermal treatments

\section{Introduction}

Rare earth (RE) doped semiconductors are interesting materials due to their applications in optoelectronics. These systems present a temperature stable luminescence wavelength which is nearly independent of the semiconductor host. The interest in RE doped semiconductors is based on the fabrication of new electroluminescence devices which can combine both the luminescence of rare earth ions and the electronic properties of semiconductors [1]. When RE elements are introduced as trivalent ions in solids they exhibit atomic-like optical transitions due to the incomplete $4 \mathrm{f}$ shell which is shielded by the outermost filled $5 \mathrm{~s}^{2}$ and $5 p^{6}$ orbitals. These intra- $4 \mathrm{f}$ transitions are parity forbidden in the free ion but are allowed when the RE ions are embedded in solids due to the mixing of opposite parity states produced by the local electric fields in the solid host [2]. In addition the RE ions weakly interact with the matrix and the wavelength of intra- $4 \mathrm{f}$ shell emission is only slightly dependent on the host material and the environmental temperature.

\footnotetext{
* Corresponding author. Tel.: + 34-91-397-4784; fax: + 34-91-3978579 .

E-mail address: jl.plaza@uam.es (J.L. Plaza)
}

RE ions have been incorporated into III-V semiconductors by doping during the growth process [3-5] opening new possibilities to construct new optoelectronic devices. $\mathrm{Er}^{3+}$ is one of the most interesting $\mathrm{RE}$ ions due to the fact that its transition from ${ }^{4} \mathrm{I}_{13 / 2}$ to the ${ }^{4} \mathrm{I}_{15 / 2}$ state appears at $1540 \mathrm{~nm}$. which is the wavelength at which the transmission loss of the silica-based optical fibbers is minimum. However, other $\mathrm{RE}$ ions as $\mathrm{Nd}$ have also been studied as optical dopants in III-V semiconductors [6,7]. This ion has shown also to be a good choice for controlling the surface charge at the semiconductor-dielectric interface [8]. In this work several changes in the properties of Er- and Nd-doped $\mathrm{GaSb}$ induced by different process of polishing, chemical etching and thermal treatment have been studied.

\section{Experimental}

Er- and Nd-doped GaSb ingots were grown by the vertical Bridgman technique. High purity $99.9999 \% \mathrm{Ga}$, $\mathrm{Sb}$ and $99.9 \% \mathrm{Er}$ and $\mathrm{Nd}$ metals were used as starting material. High quality quartz ampoules were used as crucibles and sealed at $10^{-6}$ Torr. The ampoules were also graphited by acetone pirolysis at $1000^{\circ} \mathrm{C}$ in order 
to prevent the material sticking to the walls. The growth was carried out at a rate of $3 \mathrm{~mm} / \mathrm{h}$ in a single-zone oscillatory Bridgman furnace having a temperature gradient of $35^{\circ} \mathrm{C} / \mathrm{cm}$. More details about the growth process can be found elsewhere [9]. Seven different $\mathrm{GaSb}$ ingots were grown with different dopant concentration as shown in Table 1. All of them were 60 $\mathrm{mm}$ long and $12 \mathrm{~mm}$ in diameter. The wafers obtained from the central region of the ingots were cut perpendicular to the growth direction and prepared in three different ways in order to carry out studies in (i) polished, (ii) polished and etched, and (iii) polished etched and thermally treated wafers. Along this text the samples will be referenced with the number of the ingot from which they were obtained.

Mechanical polishing was carried out using 5, 1, 0.3 and $0.05 \mu \mathrm{m}$ alumina powder. After this process, chemical etching was applied for $30 \mathrm{~s}$ with a CP4 type solution $\left(\mathrm{CH}_{3} \mathrm{COOH}: 5 \mathrm{HF}: 9 \mathrm{HNO}_{3}: 10 \mathrm{H}_{2} \mathrm{O}\right)$ followed by methanol rinsing. The thermal process was carried out after polishing and chemical etching. The wafers were placed in a quartz ampoule which was introduced in a furnace at $400^{\circ} \mathrm{C}$ for $10 \mathrm{~h}$. During this process dynamic vacuum at $10^{-6}$ Torr was established. In this way the $\mathrm{Sb}$ which is volatile at this temperature is removed permanently. In order to study the Er and $\mathrm{Nd}$ concentration in the samples, atomic absorption analysis (AAS) analysis was carried out using a Perkin-Elmer 3110 spectrometer. For the measurement of resistivity, mobility and carrier density the Van der Pauw technique at room temperature was used with magnetic fields perpendicular to the samples up to $7 \mathrm{kGauss}$. Indium dots were used as ohmic contacts which were verified previously. Secondary electron microscope (SEM) images and energy dispersive X-ray analysis (EDX) were carried out with a Philips apparatus model XL30 coupled with an EDX analyser model DX4i. The accelerating voltages were $25 \mathrm{kV}$. The cathodoluminescence (CL) data were obtained with a Hitachi S-2500 SEM at $77 \mathrm{~K}$ at $25 \mathrm{kV}$ of accelerating voltages.

\section{Results and discussion}

\subsection{Effect of the polishing and chemical etching processes}

The SEM studies of the polished surfaces showed no indications of surface damage in almost all the samples. Only the sample 27 with the highest Er concentration has shown clearly triangular precipitates of $\sim 10 \mu \mathrm{m}$ in size after the polishing process. One of these precipitates is shown in detail in Fig. 1A. The formation of these precipitates with high $\mathrm{Sb}$ and $\mathrm{Er}$ concentrations, as demonstrated below by EDX analysis, could be explained taking into account the tendency of RE elements to form compounds with $\mathrm{V}$ group elements known as pnictides [10]. The existence of precipitates has not been observed in $\mathrm{Nd}$ polished wafers even at high dopant concentration. The same kind of analysis has been carried out for polished and subsequently chemically etched wafers. Fig. 1B shows a SEM image from the surface of the pure GaSb sample (sample 24). Triangular etch pits with a density of $\sim 10^{6} \mathrm{~cm}^{-2}$ can be observed. No main differences in the surface appearance between this wafer and the samples 25, 26, 28 and 29 have been observed. Fig. 1C shows the surface of the sample 27 after polishing and chemical etching processes. In this case the precipitates shown in Fig. 1A for the polished wafers appear also. It can be observed the effect of the chemical etching which is more efficient at the boundaries. Finally, Fig. 1D shows some precipitates present in the sample 30 (highest $\mathrm{Nd}$ concentration) which could not be observed in polished wafers. This figure presents both images of secondary electron and backscattering images for two different scales.

The chemical composition of the polished wafers has been analysed for different wafers and is shown in Table 2 for pure and the highest dopant concentration. It is observed that in the pure GaSb wafer (sample 24) the content of $\mathrm{Sb}$ is $\sim 30 \%$ lower than $\mathrm{Ga}$. This deviation from the stoichiometric composition could explain the anomalous high acceptor concentration $(\sim$ $\left.10^{17} \mathrm{~cm}^{-3}\right)$ in as-grown GaSb which has been traditionally attributed to $\mathrm{Sb}$ vacants and $\mathrm{Ga}$ antisites [11]. The triangular precipitates shown in Fig. 1A have also been analysed outside and inside indicated with 'out' and 'ins' subscripts, respectively, in Table 2. These subscripts will be used along the text. It is observed that Er concentration in these precipitates is about two orders of magnitude higher inside than outside. The Sb concentration is $\sim 15 \%$ higher inside than outside while $\mathrm{Ga}$ concentration is $\sim 40 \%$ lower in the former region. The Er content outside the precipitates are very low and $\mathrm{Ga}$ and $\mathrm{Sb}$ concentrations are similar to that of the

Table 1

Initial dopant concentration $\left(\times 10^{19} \mathrm{~cm}^{-3}\right)$ for the different $\mathrm{GaSb}$ grown ingots

Ingot 24 pure Ingot $25 \mathrm{GaSb}: \mathrm{Er}$ Ingot $26 \mathrm{GaSb}: \mathrm{Er}$ Ingot $27 \mathrm{GaSb}: \mathrm{Er} \quad$ Ingot $28 \mathrm{GaSb}: \mathrm{Nd}$ Ingot $29 \mathrm{GaSb}: \mathrm{Nd}$ Ingot $30 \mathrm{GaSb}: \mathrm{Nd}$ $\mathrm{GaSb}$

1.0

2.0

8

0.6

2.4

9.6 


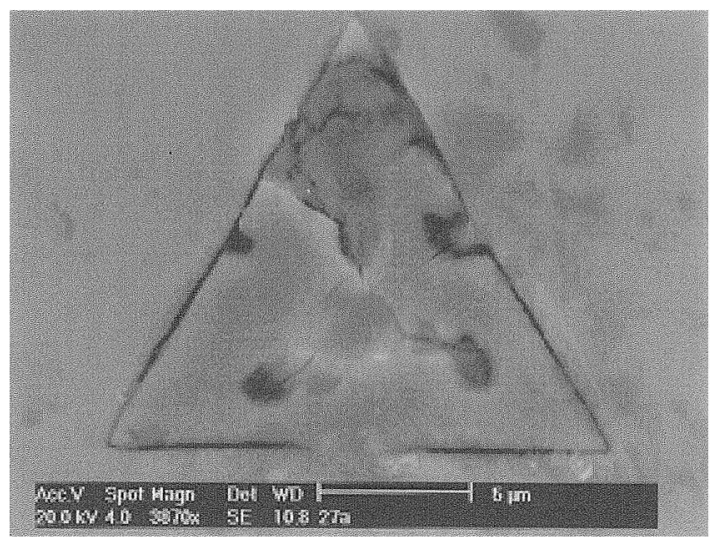

A

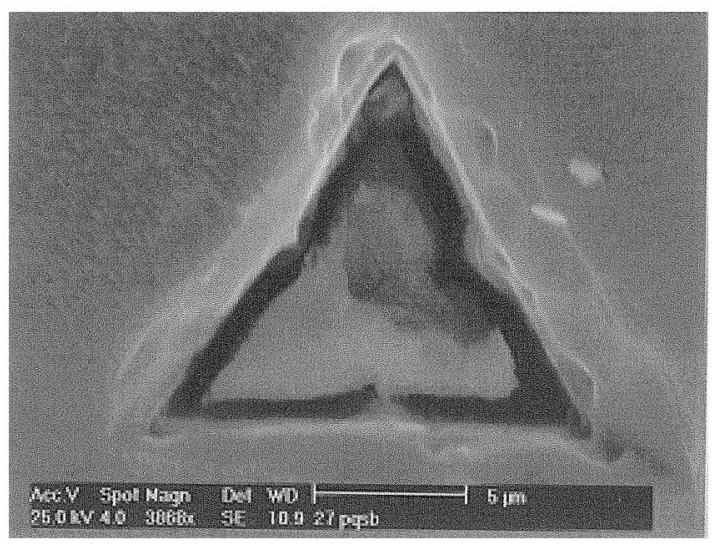

C

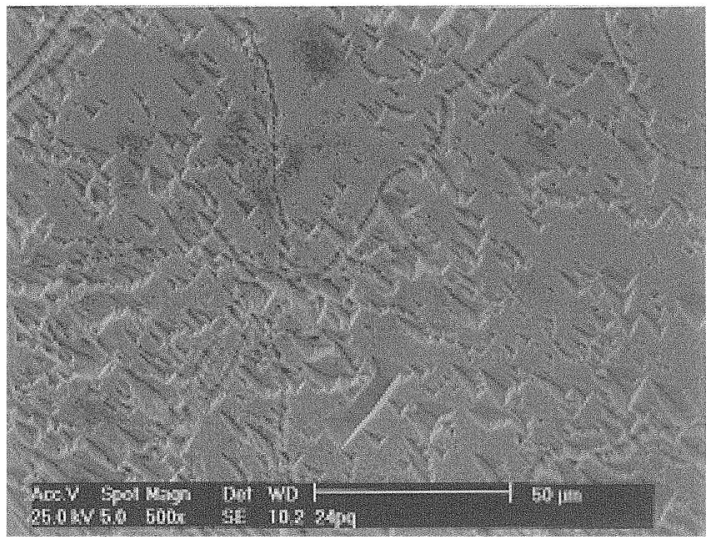

B
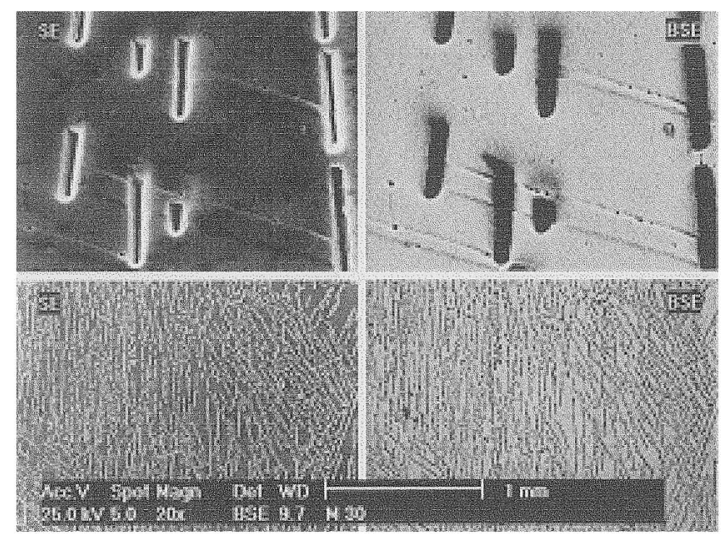

D

Fig. 1. (A) Triangular precipitates obtained from polished sample 27. (B) Image from sample 24 polished and subsequently chemically etched for $30 \mathrm{~s}$ with the etchant described in the text. (C) Detailed triangular precipitate from polished and chemically etched sample 27. (D) Two different scales in secondary electron (SE) emission and backscattering (BSE) mode images from the precipitates observed in the polished and chemically etched sample 30 .

pure GaSb. The Er concentration could not be detected for lower initial dopant concentrations.

In the case of $\mathrm{Nd}$ doped polished wafers, the concentration of $\mathrm{Nd}$ could also be analysed by EDX only for the highest doped sample 30. As shown in Table 2, the concentration of $\mathrm{Nd}$ is $\sim 0.73$ at. $\%$ on the surface. The $\mathrm{Nd}$ concentration for the two other samples grown with lower $\mathrm{Nd}$ concentrations in the melt was below the detection limit of the EDX apparatus.

For polished and subsequently chemically etched wafers, the EDX analysis performed outside and inside the etch-pits of the pure GaSb wafer presented in Fig. 1B shows that while outside the $\mathrm{Ga}$ and $\mathrm{Sb}$ concentrations are almost the same than in the case of the pure $\mathrm{GaSb}$ polished-only wafers, inside the etch pits the $\mathrm{Sb}$ concentration is $\sim 50 \%$ lower than Ga concentration. It indicates an accumulation of $\mathrm{Ga}$ in these defects. For polished and chemically etched GaSb doped with the highest Er concentration (sample 27), a slight increment
Table 2

EDAX results from different polished and chemically etched wafers

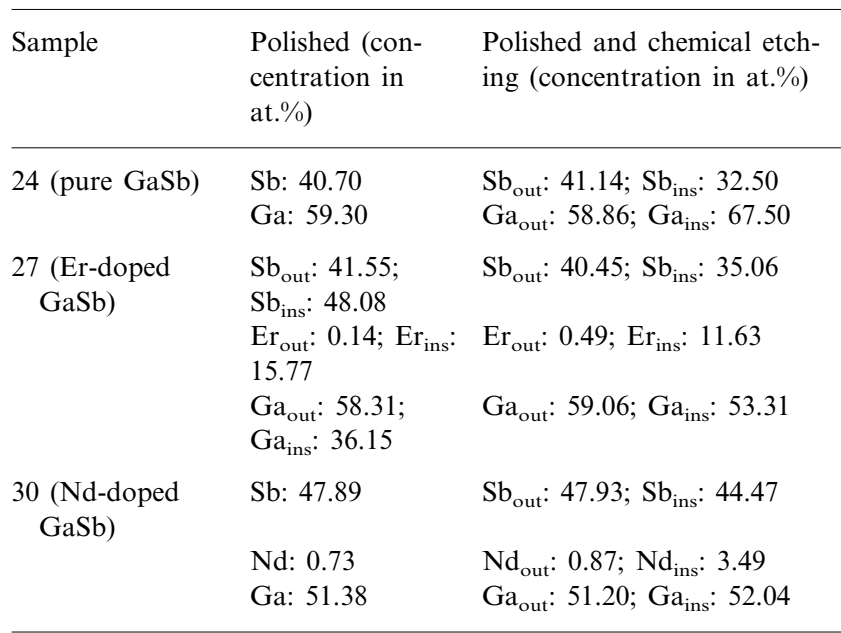




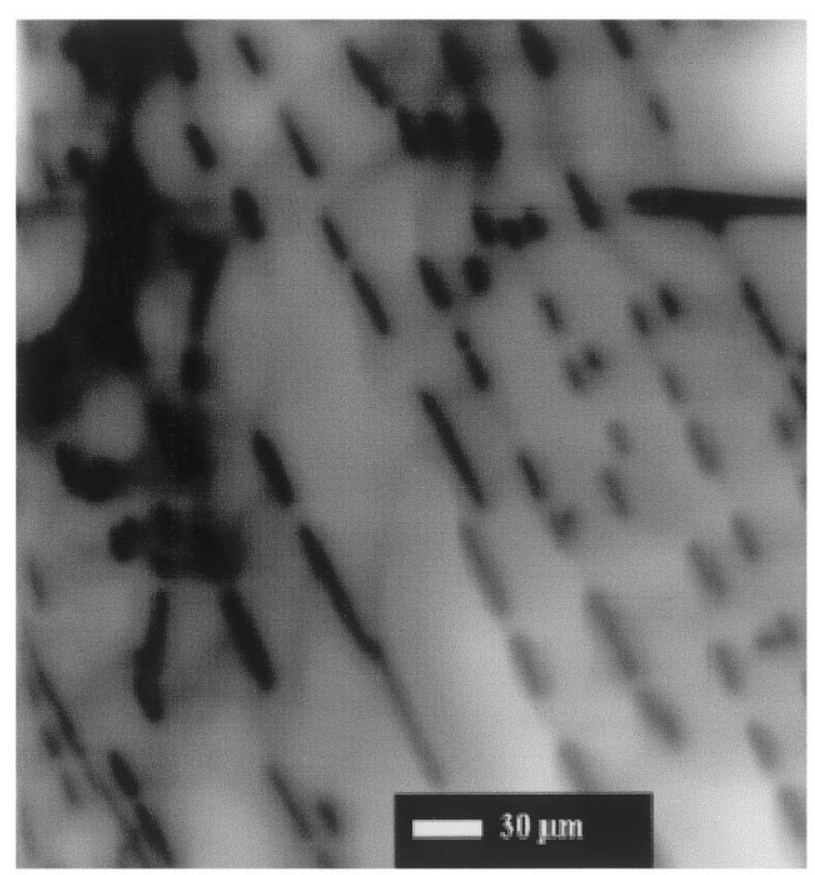

A

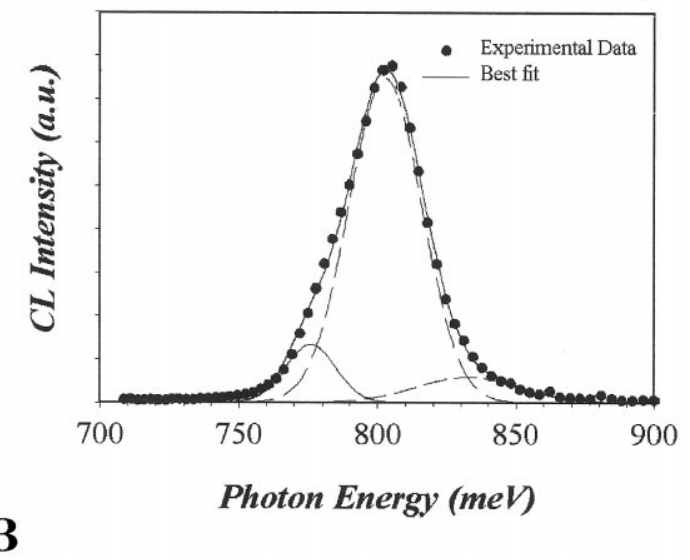

B

Fig. 2. (A) CL image and (B) CL spectrum from sample 30.

of Er corresponding to the polished wafers is observed outside the triangular precipitates as shown in Table 2. On the other hand, $\mathrm{Ga}$ and $\mathrm{Sb}$ concentrations are kept almost constant in relation to polished wafers. The EDX analysis inside has revealed a great concentration of $\mathrm{Er}$ ( $\sim 12$ at.\%). If one compares the concentrations of the three elements with that of the polished wafers, it is observed that there is a lowering of the $\mathrm{Sb}$ and $\mathrm{Er}$ concentration with an increment in the Ga concentration. It could be assumed that this is the result of a more efficient $\mathrm{Er}$ and $\mathrm{Sb}$ removal by the etchant which causes that $\mathrm{Ga}$ appears in higher concentration. EDX analysis on the elongated precipitates observed at the chemically etched surface of the sample 30 shown in Fig. 1D has been performed also in the outside and inside regions. The results shown in Table 2 present a higher (about four times) $\mathrm{Nd}$ concentration at the inside. However, the $\mathrm{Nd}$ concentration is not so high as in the case of $\mathrm{Er}$ in $\mathrm{Er}$ precipitates. $\mathrm{Ga}$ and $\mathrm{Sb}$ concentrations outside are almost the same than in the polished wafers. It must be pointed out also that in the case of $\mathrm{Nd}$ doped samples, $\mathrm{Ga}$ and $\mathrm{Sb}$ concentrations are nearer from the stoichiometric composition than in the case of pure and Er-doped $\mathrm{GaSb}$.

CL images and spectra from the three ingots have been performed to characterise the defect structure as function of the $\mathrm{Nd}$ and $\mathrm{Er}$ concentration. CL images for Er doped have been reported previously [12] where intensity of the lowest Er-doped sample 25 appeared rather uniform revealing the subgrain structure. The same structure but with smaller size of the grains has been observed in samples with higher Er-concentrations (samples 26 and 27). CL images from sample 28 reveal an uniform bright background with dark lines that correspond to grain subboundaries as reported in Ref. [13] similar to that observed in undoped material. When the Nd concentration is increased as in the sample 29, the CL images show a more inhomogeneous background. In the sample 30 elongated precipitates presented in Fig. 2A and shown previously in Fig. 1A with determined orientations and different sizes appear.

The CL spectrum for Er-doped samples have been reported previously [7]. Er doping has been found to increase the total CL intensity and to cause spectral changes. Further reduction of acceptors with increasing Er concentration has been observed in the spectrum for sample 26. However, when the Er concentration increases, the defect band A reappears. The CL spectrum from sample 28 presents the near band edge emission and the native defects related band located at $777 \mathrm{meV}$ usually observed in this material. In sample 29, with higher $\mathrm{Nd}$ concentration the defect band is detected and its intensity is comparable with the bandgap emission. For the sample 30, with the highest $\mathrm{Nd}$ concentration the spectrum shown in Fig. 2B, a marked decrease in the 777 $\mathrm{meV}$ band is achieved. This effect of reduction of native defects and enhancement in the luminescence intensity compared with other doped GaSb obtained by the Bridgman method is detected. Comparison with EDX results shows that the $\mathrm{Nd}$ effect of decreasing the defect band and increasing the band gap intensity is limited by precipitation processes at high $\mathrm{Nd}$ concentrations. 
Table 3

EDX results from different polished, chemically etched and thermally treated wafers

\begin{tabular}{ll}
\hline Sample & $\begin{array}{l}\text { Polished, chemically etched and thermally } \\
\text { treated wafers (concentration in at.\%) }\end{array}$ \\
\hline 24 (pure GaSb) & $\mathrm{Sb}: 39.03$ \\
& $\mathrm{Ga}: 60.97$ \\
$27(\mathrm{Er}$-doped & $\mathrm{Sb}_{\text {out }}: 41.93 ; \mathrm{Sb}_{\text {ins }}: 26.97$ \\
$\mathrm{GaSb})$ & $\mathrm{Er}_{\text {out }}: 0.36 ; \mathrm{Er}_{\text {ins }}: 38.60$ \\
& $\mathrm{Ga}_{\text {out }}: 57.71 ; \mathrm{Ga}_{\text {ins }}: 34.43$ \\
$30(\mathrm{Nd}-$ doped & $\mathrm{Sb}_{\text {out }}: 45.91 ; \mathrm{Sb}_{\text {ins }}: 31.47$ \\
GaSb) & $\mathrm{Nd}_{\text {out }}: 0.53 ; \mathrm{Nd}_{\text {ins }}: 18.57$ \\
& $\mathrm{Ga}_{\text {out }}: 53.56 ; \mathrm{Ga}_{\text {ins }}: 49.96$ \\
\hline
\end{tabular}

\subsection{Effect of the thermal treatment process}

In these case the wafers were polished, chemically etched and finally kept at $400^{\circ} \mathrm{C}$ in dynamic vacuum. No main differences apart from a cleaning effect were observed in the surfaces of these samples, with respect to the previously shown polished and chemically etched wafers. The EDX analysis has shown only a few changes induced by the thermal treatment in pure $\mathrm{GaSb}$ (sample 24) which presents slightly lower Sb concentration compared with the polished and chemically etched wafers probably due to $\mathrm{Sb}$ evaporation. The analysis of the samples 27 and 30 outside and inside the precipitates both of $\mathrm{Er}$ and $\mathrm{Nd}$ is shown in Table 3. However, it is worth noting that both $\mathrm{Er}$ and $\mathrm{Nd}$ concentrations have been increased inside the precipitates $\sim 50 \%$ of their values obtained from the chemically etched wafers. This behaviour could be due to the evaporation of $\mathrm{Sb}$ leaving small islands of the RE elements.

For electrical characterisation, the Van der Paw method at room temperature was used. For pure $\mathrm{GaSb}$ the values of the resistivity mobility and carrier density are shown in Table 4. The carrier density is higher for thermally treated wafers and as a result of this the resistivity and mobility are lower. This situation could be due to Sb losses which could effectively increase the $\mathrm{Ga}$ antisites.

The profiles of resistivity, mobility and carrier density versus dopant concentration are shown in Fig. 3. No appreciable changes have been observed in the electrical
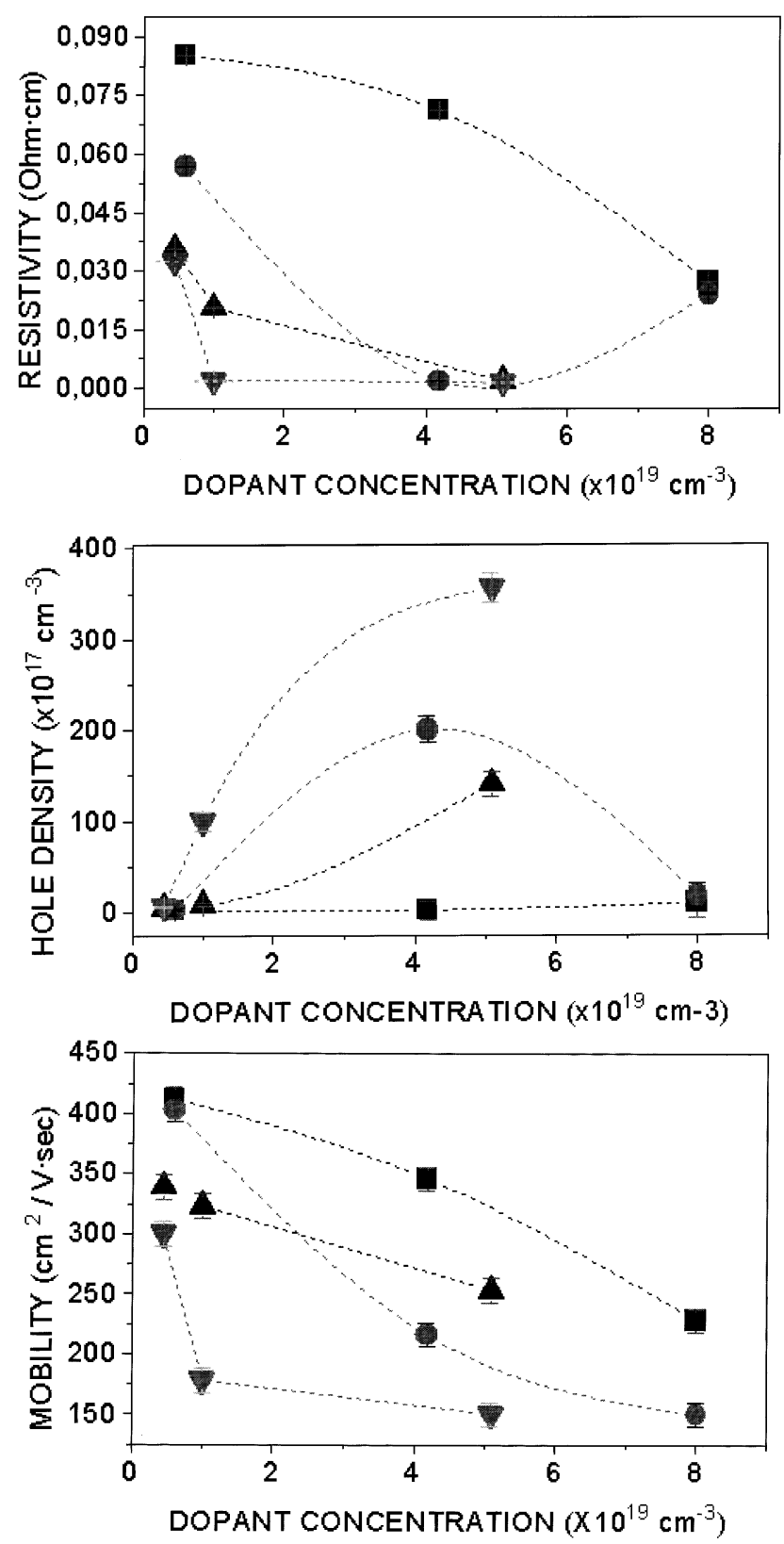

Fig. 3. (A) Resistivity, (B) carrier density and (C) mobility profiles vs. dopant concentration for Er- and Nd-doped wafers with and without annealing treatments. $\mathbf{\square}$, Nd-doped samples without annealing. $\bullet$ Nd-doped samples with annealing. $\boldsymbol{\Delta}$, Er-doped without annealing. $\boldsymbol{\nabla}$, Er-doped with annealing. The dotted lines are only shown to guide the eye.

Table 4

Resistivity, mobility and carrier density for pure GaSb (sample 24)

\begin{tabular}{|c|c|c|c|c|c|c|}
\hline Sample & $\begin{array}{l}\text { Resistivity }(\Omega \cdot \mathrm{cm}) \\
\text { No annealing }\end{array}$ & Annealing & $\begin{array}{l}\text { Mobility }\left(\mathrm{cm}^{2} /\right. \\
\text { V's })\end{array}$ & $\begin{array}{l}\text { Mobility }\left(\mathrm{cm}^{2} /\right. \\
\text { V.s }) \\
\text { Annealing }\end{array}$ & $\begin{array}{l}\text { Hole density }\left(\times 10^{17}\right. \\
\left.\mathrm{cm}^{-3}\right)\end{array}$ & $\begin{array}{l}\text { Hole density }\left(\times 10^{17}\right. \\
\left.\mathrm{cm}^{-3}\right)\end{array}$ \\
\hline
\end{tabular}


properties of polished and etched wafers so only the results obtained for thermally and no-thermally treated samples are presented. It can be observed in Fig. 3A that for low dopant concentration the resistivity decrease with the increasing dopant concentration. This behaviour is related to the increment in the carrier density which causes a diminishing mobility as shown in Fig. 3B and c, respectively. Only for the highest doped samples the resistivity rises probably as a consequence of the precipitates which could act as recombination centres.

As can be observed for similar dopant concentrations, the resistivity of the no-thermally treated $\mathrm{Nd}$ doped wafers is slightly higher than for Er-doped wafers in agreement with the higher carrier density in the later case. The resistivity is decreased due to the thermal process in all the samples as shown in Fig. 3A. This behaviour is related to the increasing carrier density as shown in Fig. 3B. This increment in the carrier density presented by the thermally treated samples is in agreement with the decreasing mobility shown in Fig. 3C. This behaviour could be explained as a consequence of the evaporation of $\mathrm{Sb}$ atoms leaving sites which could be occupied by Ga and RE atoms. Both Er and $\mathrm{Nd}$ ions act with $3+$ valence (rarely these ions present valence $2+$ ), so the increment in the hole concentration cannot be due to substitutional positions of these ions replacing the $\mathrm{Ga}$ atoms because the isoelectronic nature of $\mathrm{Ga}, \mathrm{Er}$ and $\mathrm{Nd}$ ions. In addition only interstitial positions of the RE ions in the lattice or $\mathrm{RE}_{\mathrm{Sb}}$ antisites could explain these electronic behaviour. The position of RE in the lattice is still unclear and more study is required on these features. It is worth noting that the effect of the thermal treatment related to the increasing carrier density and, as a consequence, decreasing resistivity is more pronounced in the case of the samples doped with an intermediate level of dopant (samples 26 and 29). This observation is especially noted for Er-doped sample 29 where the resistivity is about one order of magnitude higher in the case of thermally tretated sample. For the highest Er (sample 27) and $\mathrm{Nd}$ (sample 30) concentrations, the carrier density and mobility decrease probably due to precipitation resulting in a higher resistivity for these samples. As a consequence the thermal treatment has small effects in these samples.

\section{Conclusions}

In this work the formation of precipitates both of $\mathrm{Er}$ and $\mathrm{Nd}$ in $\mathrm{GaSb}$ grown by the vertical Bridgman method for an initial dopant concentration of $9 \times 10^{19} \mathrm{~cm}^{-3}$ has been demonstrated. These precipitates present a high concentration of the RE element and $\mathrm{Sb}$ compared with the surrounding material. The chemical etching also reveals a density of etch pits up to $10^{6} \mathrm{~cm}^{-2}$ which show an excess of $\mathrm{Ga}$ at the inside. The reduction of the defect band induced by the RE elements has been demonstrated by CL. This effect is diminished for high dopant concentrations due to precipitation phenomena. Electrical measurements showed that the thermal treatments increase the carrier density. This effect is more pronounced for Er- and Nd-doped samples with a dopant density of $2 \times 10^{19} \mathrm{~cm}^{-3}$.

\section{Acknowledgements}

This work has been supported by CICYT under the project ESP-98 1340.

\section{References}

[1] S. Coffa, A. Polman, R.N. Schwartz, Rare Earth Doped Semiconductors II, MRS Symposium Proceedings 422, Materials Research Society, Pittsburg, 1996.

[2] A.R. Zanatta, L.A.O. Nunes, Appl. Phys. Lett. 71 (25) (1997) 3679.

[3] L.F. Zakharenkov, V.A. Kasatkin, F.P. Kesamanly, B.E. Samorukov, M.A. Sokolova, Sov. Phys. Semicond. 15 (1981) 946.

[4] V.A. Kasatkin, F.P. Kesamanly, V.G. Makarenkov, V.F. Masterov, B.E. Samorukov, Sov. Phys. Semicond. 14 (1980) 1092.

[5] V.A. Kasatkin, F.P. Kesamanly, B.E. Samorukov, Sov. Phys. Semicond. 15 (1981) 352.

[6] H. Ennen, J. Schneider, J. Electron. Mater. 14A (1985) 115.

[7] P. Hidalgo, B. Méndez, J. Piqueras, J. Plaza, E. Diéguez, Semicond. Sci. Technol. 13 (1998) 1431.

[8] T.J. Zhang, S.Y. Li, Solid State Electron. 29 (1986) 775.

[9] P.S. Dutta, K.S. Koteswara Rao, H.L. Bhat, K. Gopalakrihna Naik, V. Kumar, J. Cryst. Growth 155 (1995) 3702.

[10] M.E. Mullen, B. Lüthi, P.S. Wang, E. Bucher, L.D. Longinotti, J.P. Maita, Phys. Rev. B 10 (1974) 1.

[11] M. Ichimura, K. Higuchi, Y. Hattori, T. Wada, N. Kitamura, J. Appl. Phys. 68 (1990) 6153.

[12] J.L. Plaza, P. Hidalgo, B. Méndez, J. Piqueras, J.L. Castaño, E. Diéguez, J. Cryst. Growth 198 (1999) 379.

[13] B. Méndez, J. Piqueras, P.S. Dutta, E. Diéguez, Appl. Phys. Lett. 67 (1995) 2648. 